\title{
AVALIAÇÃO E MAPEAMENTO DOS SERVIÇOS ECOSSISTÊMICOS OFERTADOS PELA RESERVA BIOLÓGICA NASCENTES DA SERRA DO CACHIMBO, PARÁ, BRASIL
}

\author{
Mayara Gomes da Silva ${ }^{1}$ \\ Norma Ely Santos Beltrão² \\ Gundisalvo Piratoba Morales ${ }^{3}$
}

Resumo: A identificação, classificação e mapeamento de serviços ecossistêmicos fornecem uma visão geral dos ecossistemas, além de salientar a relevância da biodiversidade e funcionamento dos ecossistemas de forma eficaz. $O$ presente estudo objetivou identificar os SEs e localizá-los espacialmente na Reserva Biológica Nascentes da Serra do Cachimbo considerando as condições e classes de uso da terra observadas no ano de 2017. A metodologia escolhida baseou-se no estudo de Burkhard et al. (2009) e os resultados encontrados mostraram um alto potencial de oferta na reserva, principalmente em áreas naturais e a perda no fornecimento de serviços de regulação e culturais em áreas alteradas. Conclui-se que a área tem seus serviços ecossistêmicos ameaçados e necessitam de atenção para evitar maior comprometimento da dinâmica natural na reserva.

Palavras-chave: Unidades de Conservação. Uso do solo. Capital Natural.

\section{ASSESSING AND MAPPING ECOSYSTEM SERVICES SUPPLY BY BIOLOGICAL RESERVE NASCENTES DA SERRA DO CACHIMBRO, PARÁ, BRAZIL}

Abstract: Identifying, classifying and mapping the ecosystem services provide an overview of ecosystems, as well as highlight the relevance of biodiversity and of their effective functioning. This study aimed to identify the ES and locate them spatially in the Nascentes da Serra do Cachimbo Biological Reserve considering the conditions and land use classes observed in 2017. The chosen methodology was based on the study by Burkhard et al. (2009) and the results showed a high supply potential in the reserve, especially in natural areas and the loss of provision of cultural and regulatory services. It is concluded that the area has its ecosystem services threatened and need attention to avoid further compromise of the natural dynamics in the reserve.

Keywords: Conservation Units. Land Use. Natural Capital.

\section{EVALUACIÓN Y MAPEO DE LOS SERVICIOS ECOSISTÉMICOS QUE OFRECE LA RESERVA BIOLÓGICA NACIDA DE SERRA DO CACHIMBO, PARÁ, BRASIL}

Resumen: La identificación, clasificación y mapeo de los servicios de los ecosistemas proporciona una visión general de los ecosistemas, además de resaltar la relevancia de la biodiversidad y el funcionamiento de los ecosistemas de manera

\footnotetext{
1 Universidade do Estado do Pará, Programa de Pós-Graduação em Ciências Ambientais, Belém PA, Brasil, mayara_gomes12@hotmail.com, https://orcid.org/0000-0001-6889-4088

2 Universidade do Estado do Pará, Centro de Ciências Naturais e Tecnologia, Belém - PA, Brasil, normaelybeltrao@gmail.com, https://orcid.org/0000-0003-1991-2977

${ }^{3}$ Universidade do Estado do Pará, Centro de Ciências Naturais e Tecnologia, Belém - PA, Brasil, gundymorales@yahoo.com.br, https://orcid.org/0000-0002-5930-7053
} 
efectiva. El presente estudio tuvo como objetivo identificar las SE y ubicarlas espacialmente en la Reserva Biológica Nascentes da Serra do Cachimbo considerando las condiciones y clases de uso del suelo observadas en el año 2017. La metodología elegida se basó en el estudio de Burkhard et al. (2009) y los resultados encontrados mostraron un alto potencial de oferta en la reserva, principalmente en áreas naturales y la pérdida en la provisión de regulación y servicios culturales en áreas alteradas. Se concluye que el área tiene sus servicios ecosistémicos amenazados y necesita atención para evitar un mayor deterioro de la dinámica natural en la reserva.

Palabras clave: Unidades de conservación. Uso del suelo. Capital natural.

\section{Introdução}

O capital natural é uma fonte vital para a provisão de diversos serviços ecossistêmicos - SEs, os quais podem ser entendidos como características, funções ou processos ecológicos que direta ou indiretamente contribuem para o bem-estar humano (CONSTANZA et al., 1997; DAILY, 1997; MILLENNIUM ECOSYSTEM ASSESSMENT - MEA, 2005). A provisão de água e alimentos, regulação climática, manutenção da diversidade genética, opções de recreação, entre outros, são exemplos de benefícios fornecidos pelo meio ambiente (DAILY, 1997; LIU et al., 2016; CRESPIN; SIMONETTI, 2016).

A identificação e a classificação dos serviços ecossistêmicos fornecem uma visão geral das principais funções e produtos atribuídos aos ecossistemas, tal como suas estruturas ecológicas e processos associados (DE GROOT et al., 2002). Além disso, a identificação dos SEs contribui para reforçar acerca da relevância da biodiversidade e funcionamento dos ecossistemas, e auxilia na alocação eficientes dos recursos naturais. (DE GROOT et al., 2010; ALKEMADE et al., 2014; HEAL et al., 2005).

Dessa forma, o mapeamento de serviços ecossistêmicos vem ganhando notoriedade e tem sido cada vez mais utilizado para fornecer informações na tomada de decisão, auxiliando no aumento da conscientização da população e na criação de estratégias para conservação, visto que a perda de SEs implica na perda de benefícios da natureza ao homem, que em muitos casos, impacta os sistemas sociais e econômicos (BURKHARD et al., 2013; BURKHARD; MAES, 2017; CROSSMAN et al., 2013; WOLFF et al., 2015; MALINGA et al., 2015; MAES et al., 2011). Além do mapeamento, a avaliação principalmente espacialmente de SEs auxiliam na integração desses serviços no planejamento, gerenciamento e tomada de decisão, visto que informam aos gestores sobre a dinâmica dos SEs em 
determinada paisagem (BARÓ et al., 2016; GEIJZENDORFFER et al., 2015). No que tange às florestas tropicais, diversos estudos vêm desenvolvendo 0 mapeamento e avaliação de SEs (MILHEIRAS; MACE, 2019; LE CLEC'H et al., 2018; RAMIREZ-GOMEZ et al., 2015).

Especificamente a identificação, mapeamento e avaliação dos serviços ofertados pelas florestas tropicais se diferem entre os vários métodos existentes, como utilização de ferramentas de mapeamento participativo, mapeamento de SEs de provisão a partir de indicadores selecionados, entre outros (RAMIREZ-GOMEZ et al., 2015; LE CLEC'H et al., 2018). Sendo assim, observa-se o uso de mapas como um dos elementos essenciais para visualização desses serviços possibilitando sua espacialização no tempo e espaço, identificando áreas que devem ser conservadas devido à grande oferta de SEs (KANDZIORA et al., 2013; TROY; WILSON, 2006; BALVANERA et al., 2001).

Nessa perspectiva, Burkhard et al. (2009) propuseram um método de mapeamento que associa a opinião de profissionais sobre SEs e ferramentas de geoprocessamento na elaboração da matriz de SEs e mapas temáticos (SOHEL et al., 2015). Esse mapeamento pode revelar consequências decorrentes das mudanças do uso e ocupação do solo, que ocorrem através de atividades antrópicas e geram efeitos nos ecossistemas naturais, afetando diretamente a oferta dos SEs (KAUSHAL et al., 2017; CROSSMAN et al., 2012).

$\mathrm{Na}$ tentativa de garantir a oferta de serviços ecossistêmicos, as Unidades de Conservação - UCs são espaços territoriais com características naturais relevantes, legalmente instituídas pelo Poder Público (BRASIL, 2000). Essas áreas além protegerem a diversidade biológica, funcionam como fonte de SEs, que beneficia direta ou indiretamente as sociedades humanas, em particular as que estão mais próximas a essas áreas com a provisão de água e alimentos, manutenção da biodiversidade, regulação climática, proteção do solo, outros (YOUNG; MEDEIROS, 2018).

Apesar da grande importância da criação e manutenção das UCs na região Amazônica, elas estão sob constante pressão através de atividades agropecuárias, exploração ilegal madeireira, entre outros (BAIMA et al., 2018). Tais atividades quando desenvolvidas dentro de áreas protegidas, contribuem para 0 desmatamento, considerada a principal perda dos SEs na Amazônia brasileira (CASTRO; ANDRADE, 2016). 
O estado do Pará é o 2ํㅡ maior estado da região Amazônica em área territorial, e também o que possui mais áreas designadas para UCs federais (KZAM et al., 2018; INSTITUTO SOCIOAMBIENTAL - ISA, 2019). Como recorte amostral para entendimento deste contexto, tem-se a Reserva Biológica Nascentes da Serra do Cachimbo - RBNSC, unidade de conservação de proteção integral, que em 2018 figurou como a $12^{\mathrm{a}}$ UC mais desmatada da Amazônia, onde a exploração madeireira e pecuária são apontados como as principais causas dessas alterações (BAIMA et al., 2018; BRASIL, 2009), e apesar dos dados mostrarem expansão da degradação nessa UC, ainda são escassos os estudos sobre a dinâmica do uso da terra na Rebio.

Tendo em vista o crescente processo de degradação florestal e a perda de serviços ecossistêmicos, faz-se necessário compreender o impacto da dinâmica de uso da terra na Rebio em estudo na oferta de SEs. Para alcançar esse objetivo propõe-se nesta pesquisa, identificar o potencial de oferta de SEs e localizá-los espacialmente na Reserva Biológica Nascentes da Serra do Cachimbo considerando as condições e classes de uso da terra observadas no ano de 2017. Ao associar classes de uso com os SEs intrínsecos, espera-se reforçar a importância da área protegida dados as perdas dos benefícios ofertados pelas florestas e seus impactos nos sistemas humanos e naturais.

\section{Área de estudo}

A RBNSC está localizada no sul do Pará, entre as coordenadas geográficas

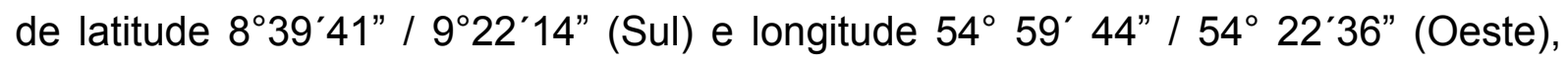
abrangendo parte dos municípios de Altamira e Novo Progresso, adjacente à BR163, conforme Figura 1 (BRASIL, 2009). A reserva, pertencente à categoria de proteção integral, foi criada pelo Decreto de 20 de maio de 2005, com o objetivo de preservar os ecossistemas naturais existentes, possibilitando a realização de pesquisas científicas e o desenvolvimento de atividades controladas de educação ambiental (BRASIL, 2005). 
Figura 1 - Mapa de localização da RBNSC.

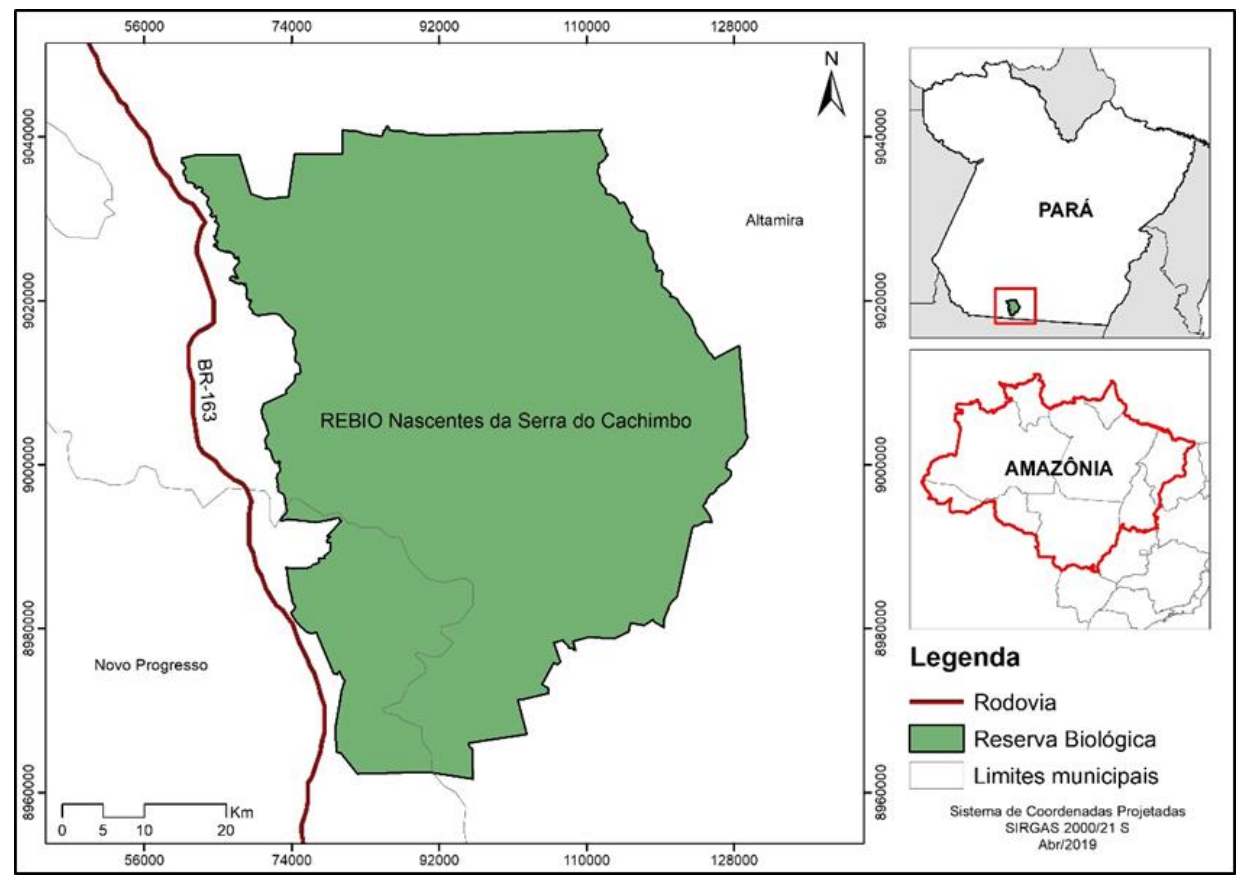

Fonte: IBGE (2017); ISA (2017).

Conforme o mapeamento descrito no Plano de Manejo da unidade, a área apresenta um mosaico de formações vegetais, composto sobretudo de Floresta Ombrófila Sub-Montana e Campinaranas, além de pequenas manchas de Floresta Estacional e áreas antropogênicas (BRASIL, 2009). O clima varia entre o equatorial úmido e o tropical, Af e Aw na classificação de Köppen respectivamente (BRASIL, 2009). A pluviosidade apresenta-se variável, entre $1800 \mathrm{~mm}$ a $2200 \mathrm{~mm}$ por ano e a temperatura média anual de $22^{\circ} \mathrm{C}$ (BRASIL, 2009; CADASTRO NACIONAL DE UNIDADES DE CONSERVAÇÃO - CNUC, 2019).

\section{Material e métodos}

\section{Coleta e análise de dados}

A metodologia escolhida baseou-se no estudo de Burkhard et al. (2009) utilizando procedimentos importantes para atingir o objetivo da pesquisa, como escolha da área de estudo e da base de dados para análise espacial do uso da terra, levantamento bibliográfico e documental, identificação dos SEs e atribuição de pesos, revisão dos especialistas sobre os pesos de cada serviço associado à classe e uso de técnicas de geoprocessamento para mapear e espacializar as informações, conforme descrito na Figura 2. 
Figura 2 - Procedimentos realizados para o desenvolvimento da pesquisa.

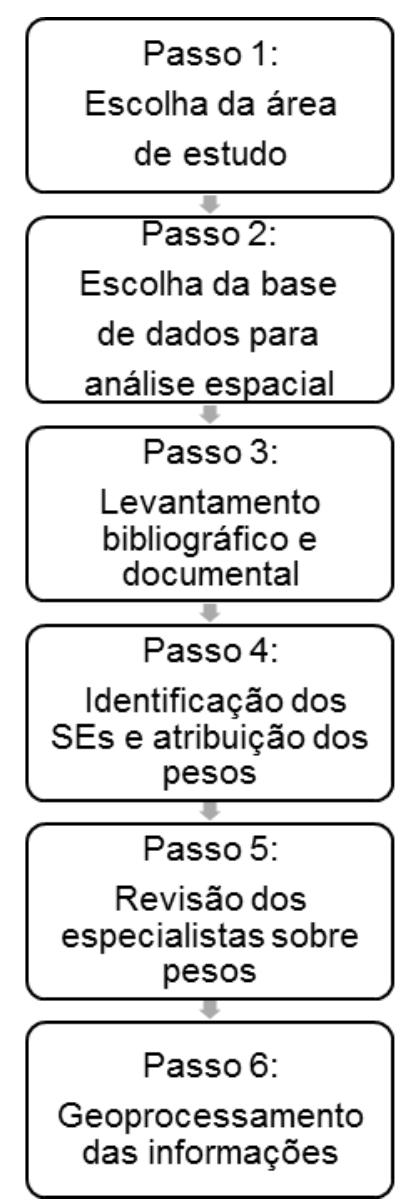

Fonte: Elaboração própria, 2019/Adaptado de Zhang e Ramírez (2019).

A análise do uso do solo baseou-se na classificação realizada pelo Projeto de Mapeamento Anual da Cobertura e Uso do Solo do Brasil - MapBiomas. O MapBiomas é um projeto que utiliza processamento em nuvem e classificadores automatizados de imagens capturadas pelo satélite Landsat, desenvolvidos e operados a partir da plataforma Google Earth Engine - GEE para todo o território do Brasil (MAPBIOMAS, 2019).

Foi utilizada a imagem classificada referente ao ano de 2017, último ano mapeado pelo projeto. O mapeamento foi realizado em uma escala de 1:250.000, o qual foi extraído pela plataforma do GEE e processado no software ArcMap 10.5. Foram identificadas seis diferentes classes de uso: Formação Florestal; Outra Formação Natural Não Florestal; Agropecuária; Outra Área Não Vegetada; Rio, Lago e Oceano; Não Observado, porém, a escala utilizada impossibilitou detalhe suficiente para identificação de duas delas, "outra área não vegetada" e "não observado", por conta disso, não constam no mapa. As classes identificadas foram 
usadas para determinar, qual a capacidade de determinada classe em fornecer os SEs, baseado no estudo de Burkhard et al. (2013).

Utilizou-se a classificação apresentada pelo projeto The Economics of Ecosystems and Biodiversity - TEEB (2010) para a identificação dos serviços oferecidos pela Rebio, que propôs 22 serviços, dentro de 4 diferentes categorias: serviços de provisão, de regulação, de manutenção e culturais, conforme descrito no Quadro 1. Essa classificação, segundo Gómez-Baggethun e Barton (2013), foi baseada em categorizações anteriores apresentadas por Daily (1997), de Groot et al. (2002) e MEA (2003).

Quadro 1 - Classificação dos SEs

\begin{tabular}{|c|c|c|}
\hline Categorias & № & Serviços Ecossistêmicos \\
\hline \multirow{6}{*}{ Provisão } & 1 & Provisão de alimentos \\
\hline & 2 & Fornecimento de água \\
\hline & 3 & Matéria-prima \\
\hline & 4 & Recursos genéticas \\
\hline & 5 & Recursos medicinais \\
\hline & 6 & Recursos ornamentais \\
\hline \multirow{9}{*}{ Regulação } & 7 & Regulação da qualidade do ar \\
\hline & 8 & Regulação climática \\
\hline & 9 & Moderação de eventos extremos \\
\hline & 10 & Regulação do fluxo de água \\
\hline & 11 & Tratamento de efluentes \\
\hline & 12 & Prevenção de erosão \\
\hline & 13 & Ciclagem de nutrientes \\
\hline & 14 & Polinização \\
\hline & 15 & Controle Biológico \\
\hline \multirow{2}{*}{ Manutenção } & 16 & Manutenção da diversidade genética \\
\hline & 17 & Habitat para espécies \\
\hline
\end{tabular}




\begin{tabular}{|l|l|l|}
\multirow{4}{*}{ Culturais } & 18 & Informação estética \\
\cline { 2 - 3 } & 19 & Recreação \\
\cline { 2 - 3 } & 20 & Inspiração para cultura, arte e design \\
\cline { 2 - 3 } & 21 & Experiência espiritual \\
\cline { 2 - 3 } & 22 & Desenvolvimento cognitivo \\
\hline \multicolumn{3}{|c|}{ Fonte: Adaptado de TEEB (2010). } \\
\hline
\end{tabular}

A metodologia, baseada no estudo Burkhard et al. (2009), consistiu na atribuição de pesos aos SEs de acordo com a capacidade da classe de uso em prover determinado serviço e na opinião de especialistas da área, configurando uma análise subjetiva, realizado também em estudos de Zhang e Ramírez (2019), Depellegrin et al. (2016) e Sohel et al. (2015). A atribuição dos pesos foi realizada durante os meses de maio e junho de 2019 , onde três especialistas com formações relacionadas ao meio ambiente - engenheiros florestais e engenheiro ambiental, contribuíram para a determinação dos pesos relacionando as classes de uso com os SEs. Logo, os pesos variavam em uma escala de 0 a 5 , onde 0 representava sem capacidade de prover o serviço, 1 baixa relevância, 2 alguma capacidade relevante, 3 média relevância, 4 alta relevância e 5 muito alta relevância em prover o SE, onde profissionais avaliaram individualmente e posteriormente foi atribuído o peso que estava em concordância com a opinião do grupo de especialistas, em conformidade com o método usado por Zhang e Ramírez (2019).

A partir dos resultados obtidos com a aplicação de atribuição de pesos, foram elaborados primeiramente quatro mapas temáticos para demonstrar o potencial de oferta dos serviços ecossistêmicos de cada uma das quatro categorias: provisão, regulação, manutenção e culturais. A partir de então, utilizou-se o processo de álgebra de mapas no software ArcGis 10.5, para representar de uma forma geral a oferta de todos os SEs nessa área. Além disso, elaborou-se quadros e gráficos no software Microsoft Office Excel 2016 para melhor representação dos resultados.

\section{Resultados e discussão}

Figura 3 mostra o mapa temático em relação às classes de uso da terra na RBNSC, onde apresenta principalmente "formação florestal" e "formação natural não 
florestal", caracterizadas como floresta ombrófila e campinarana, respectivamente. Por outro lado, podem ser observadas áreas representativas de atividade agrícola e pecuária no interior da UC, próximo de áreas de floresta densa e a classe "rio, lago e oceano" referente aos corpos hídricos.

Figura 3 - Mapa de uso e ocupação do solo referente ao ano de 2017.

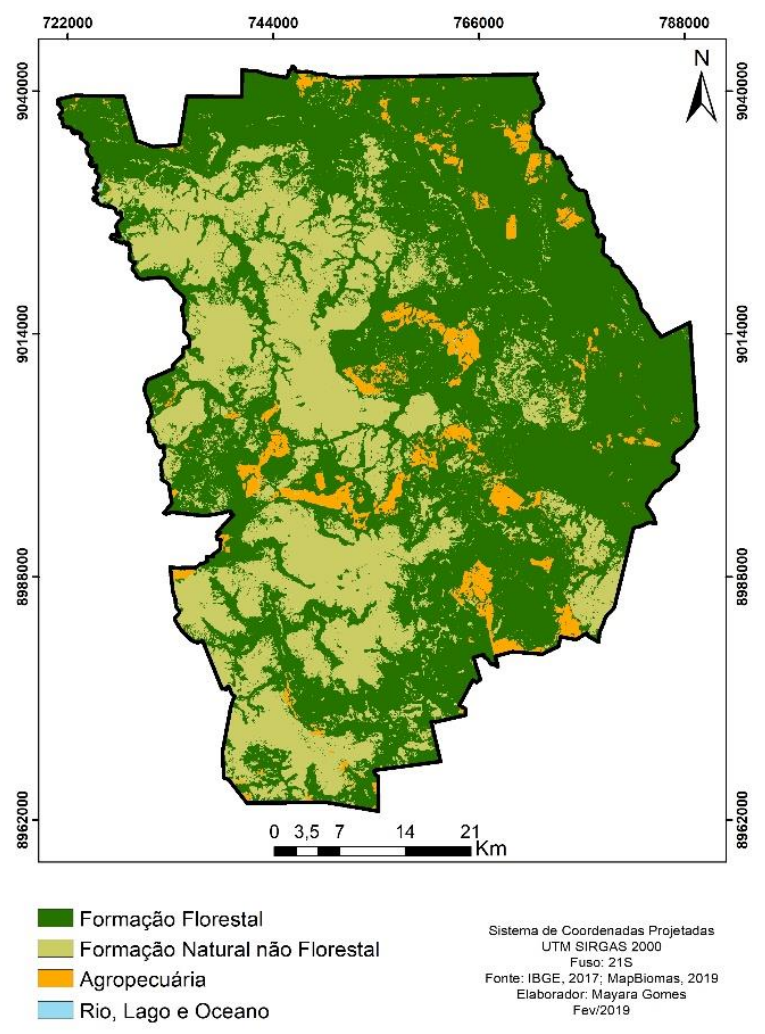

Fonte: Autores, 2019.

A partir do mapa de uso e ocupação da terra, pôde-se atribuir pesos para os SEs de acordo com as classes utilizadas na análise, "Formação florestal", "Outra formação natural não florestal", "Agropecuária" e "Rio, lago e oceano", variando em uma escala de 0 a 5, conforme descrito no Quadro 2. As duas classes que não constam no mapa devido ao nível de detalhes da escala utilizada, também não apresentaram potencial de oferta de serviços ecossistêmicos pelo fato de serem áreas com predomínio de estradas e construções (sem vegetação) e áreas não identificadas, como "outra área não vegetada" e "não observado", respectivamente, obtendo peso $0 \mathrm{em}$ todos os serviços ecossistêmicos. Dentre as demais, "formação florestal" e "formação natural não florestal" foram as que obtiveram maiores pesos, de acordo com a expertise dos especialistas, por apresentarem maior oferta de SEs. 
Quadro 2 - Matriz de avaliação de serviços ecossistêmicos de acordo as respectivas classes de uso da terra.

\begin{tabular}{|c|c|c|c|c|c|c|}
\hline SEs & $\begin{array}{r}\text { Formação } \\
\text { Florestal }\end{array}$ & $\begin{array}{l}\text { Formação Natural } \\
\text { não Florestal }\end{array}$ & Agropecuária & $\begin{array}{c}\text { Outra área não } \\
\text { vegetada }\end{array}$ & $\begin{array}{c}\text { Rio, Lago e } \\
\text { Oceano }\end{array}$ & $\begin{array}{c}\text { Não } \\
\text { observado }\end{array}$ \\
\hline \multicolumn{7}{|l|}{ Provisão } \\
\hline Provisão de alimentos & 5 & 3 & 5 & 0 & 4 & 0 \\
\hline Fornecimento de água & 0 & 0 & 0 & 0 & 5 & 0 \\
\hline Matéria-prima & 5 & 3 & 5 & 0 & 2 & 0 \\
\hline Recursos genéticas & 5 & 4 & 2 & 0 & 5 & 0 \\
\hline Recursos medicinais & 5 & 4 & 2 & 0 & 2 & 0 \\
\hline Recursos ornamentais & 5 & 3 & 0 & 0 & 1 & 0 \\
\hline \multicolumn{7}{|l|}{ Regulação } \\
\hline Regulação da qualidade do ar & 5 & 3 & 1 & 0 & 3 & 0 \\
\hline Regulação climática & 5 & 3 & 1 & 0 & 3 & 0 \\
\hline $\begin{array}{c}\text { Moderação de eventos } \\
\text { extremos }\end{array}$ & 4 & 3 & 0 & 0 & 0 & 0 \\
\hline Regulação do fluxo de água & 5 & 3 & 0 & 0 & 5 & 0 \\
\hline Tratamento de efluentes & 0 & 0 & 0 & 0 & 4 & 0 \\
\hline Prevenção de erosão & 4 & 3 & 0 & 0 & 0 & 0 \\
\hline Ciclagem de nutrientes & 5 & 2 & 0 & 0 & 1 & 0 \\
\hline Polinização & 5 & 4 & 0 & 0 & 0 & 0 \\
\hline Controle Biológico & 5 & 5 & 2 & 0 & 3 & 0 \\
\hline \multicolumn{7}{|l|}{ Manutenção } \\
\hline $\begin{array}{c}\text { Manutenção da diversidade } \\
\text { genética }\end{array}$ & 5 & 5 & 2 & 0 & 5 & 0 \\
\hline Habitat para espécies & 5 & 5 & 2 & 0 & 4 & 0 \\
\hline \multicolumn{7}{|l|}{ Culturais } \\
\hline Informação estética & 5 & 2 & 1 & 0 & 5 & 0 \\
\hline Recreação & 3 & 0 & 0 & 0 & 3 & 0 \\
\hline $\begin{array}{c}\text { Inspiração para cultura, arte e } \\
\text { design }\end{array}$ & 4 & 3 & 1 & 0 & 4 & 0 \\
\hline Experiênia espiritual & 4 & 3 & 0 & 0 & 4 & 0 \\
\hline Desenvolvimento cognitivo & 4 & 3 & 0 & 0 & 4 & 0 \\
\hline
\end{tabular}

Pesos variam de 0 a 5, representando a capacidade da classe em prover o SE. 0: sem capacidade; 1 : baixa relevância; 2: alguma capacidade relevante; 3: média relevância; 4: alta relevância; 5: muito alta relevância.

Fonte: Autores, 2019.

A matriz de avaliação dos SEs resultou em um gráfico de radar para verificação da oferta de cada categoria: provisão, regulação, manutenção e culturais, considerando apenas as classes de uso da terra que obtiveram pesos acima de 0 na análise, de acordo com a Figura 4. Sendo assim, observa-se a relevante contribuição da "formação florestal", "formação natural não florestal" e "rio, lado e oceano", como áreas naturais com significativo potencial de fornecer serviços de diferentes categorias. Por outro lado, verifica-se que a classe "agropecuária", área antropizada, está basicamente resumida em dispor serviços de provisão e de manutenção. 
Figura 4 - Gráfico da oferta de SEs em cada categoria

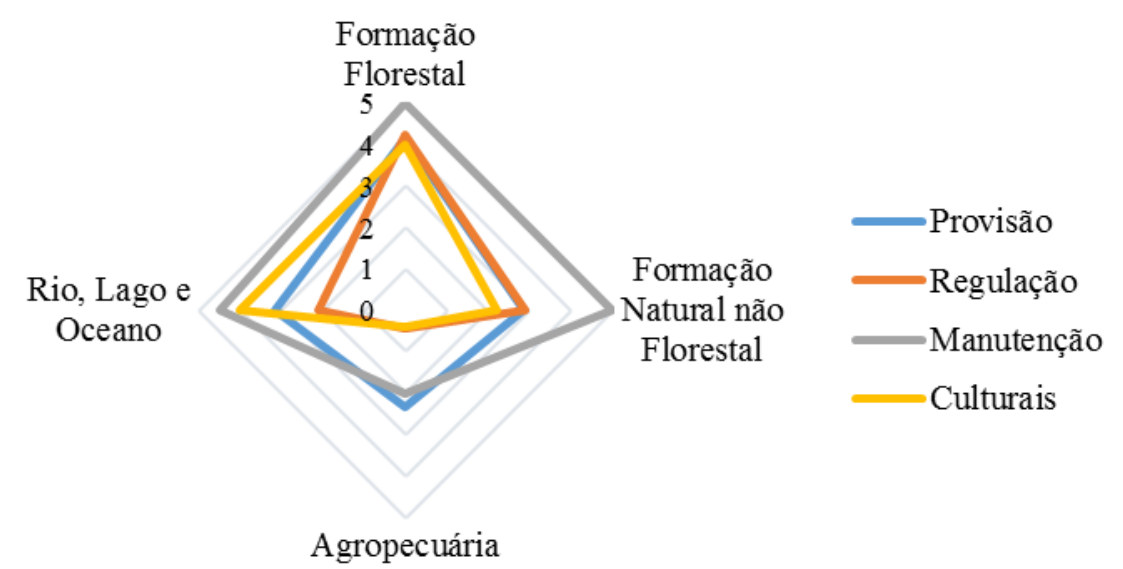

Fonte: Autores, 2019.

Conforme observado na Figura 5, os serviços ecossistêmicos foram espacializados na área de estudo para identificação em cada categoria de SE, onde possui maior potencial de oferta, seguindo as notas atribuídas pelos especialistas anteriormente. De uma forma geral, percebe-se que apenas as áreas construídas e desflorestadas não possuem ou possuem baixa capacidade de ofertar serviços, o restante da área da Rebio apresenta pelo menos alguma capacidade relevante de fornecimento de algum serviço.

A combinação dos quatro mapas gerou o mapa geral da oferta desses serviços, disponível na Figura 6. Este mapa demonstra a disponibilidade e oferta dos serviços ecossistêmicos na Reserva Biológica Nascentes da Serra do Cachimbo. Dessa forma, observa-se que prioritariamente a alta capacidade de oferta dos SEs concentra-se nas áreas naturais, especialmente áreas de vegetação densa, seguido das campinaranas, e são mais escassos em áreas alteradas pelo homem, levando à baixa capacidade de fornecer bens e serviços dos ecossistemas. 
Figura 5 - Mapas da distribuição espacial da oferta de SEs
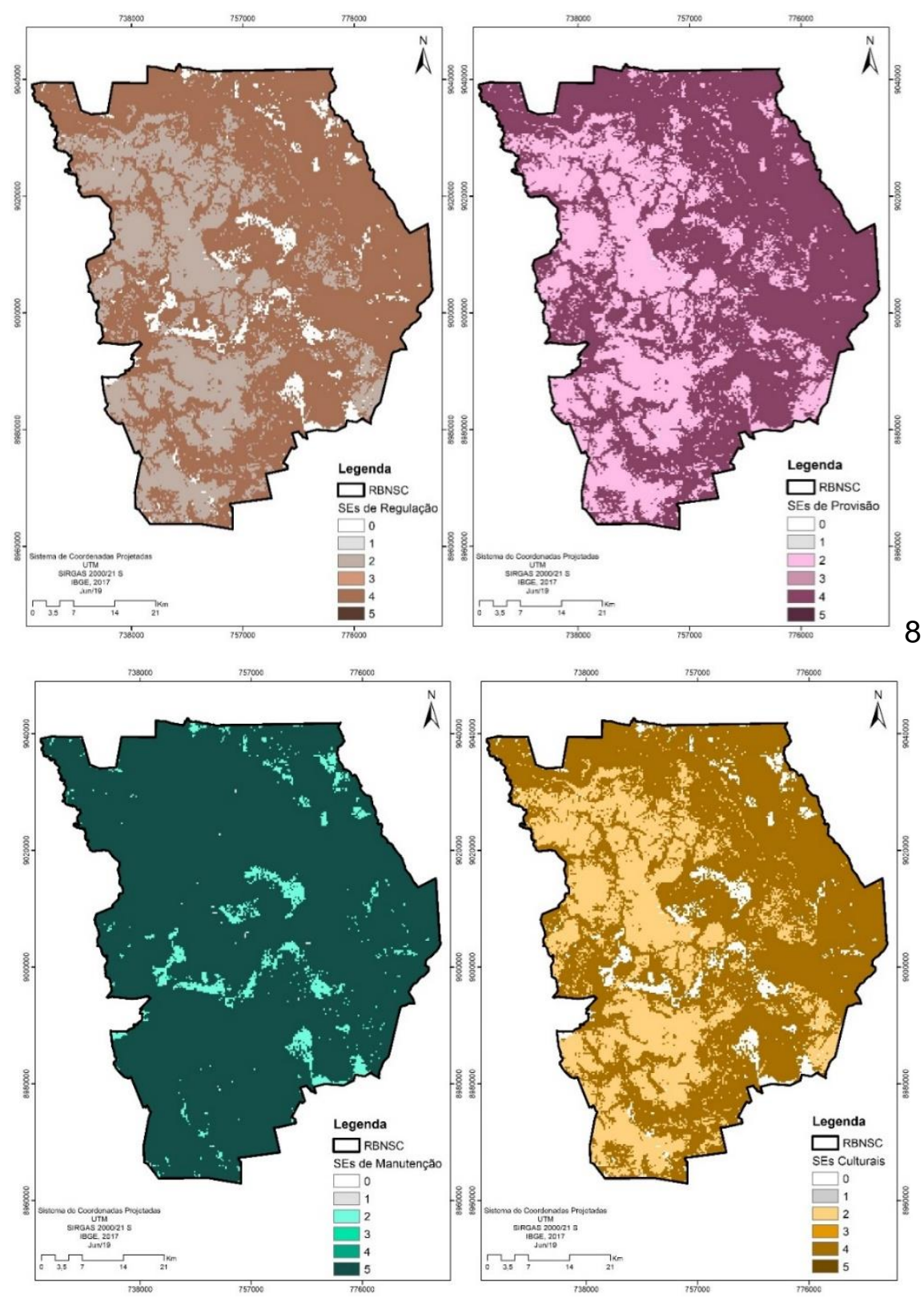

Fonte: Autores, 2019. 
Figura 6 - Mapa da oferta de SEs na RBNSC

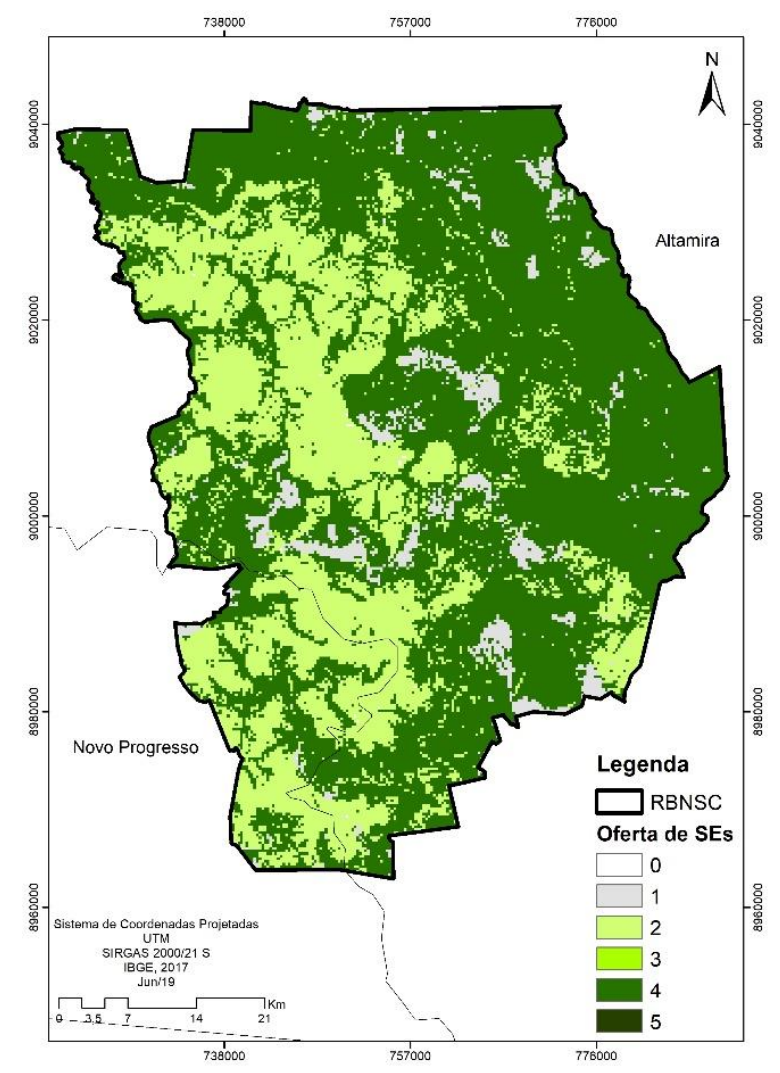

Fonte: Autores, 2019.

Considerando os resultados encontrados, observou-se uma presença significativa de áreas na cor cinza à leste da UC, ou seja, indicando um menor potencial na oferta de serviços ecossistêmicos na região pertencente ao município de Altamira. O município de Altamira foi uma das áreas centrais do Programa Integrado de Colonização - PIC, que estimulava atividades agrícolas para o mercado, levando a mudanças consideráveis nas formas do uso da terra para acompanhar as transformações socioeconômicas no município (SILVA et al., 2017; SILVA et al., 2015). Além de Altamira, Novo Progresso é um município que também se destaca entre os que mais desmatam no estado do Pará (TUPIASSU et al., 2018), em vista disso, das 10 UCs mais desmatadas da Amazônia, 3 das 4 localizadas no Pará estão localizadas entre Novo Progresso e Altamira, são elas:

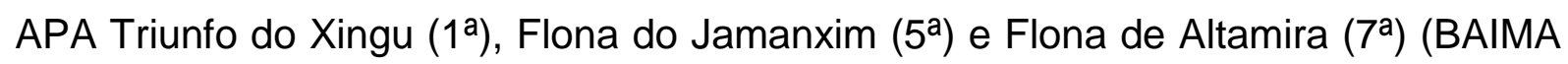
et al., 2018).

Segundo Monteiro (2018) e Dal'Aasta et al. (2012), Novo Progresso e Altamira fazem parte do Distrito Florestal Sustentável - DFS, criado em 2006 a partir do Projeto BR-163. Dentre as ações propostas no DFS, a criação e efetivação de unidades de conservação nesses municípios eram uma delas, como forma de 
reduzir impactos socioambientais nessas áreas, principalmente as taxas de desmatamento e atividade madeireira ilegal, no entanto, entre 2000 a 2010 Novo Progresso e Altamira mantiveram com os maiores índices de desflorestamento do DSF, aproximadamente $8.300 \mathrm{~km}^{2}$, tornando-os munícipios com intensa pressão.

Dados do estudo de Baima et al. (2018) afirmam que dentro da região amazônica, o estado do Pará foi o que mais desflorestou em unidades de conservação federais entre 2012 e 2017, quase 92 mil hectares. Fato este que está relacionado com a região do estado situada no Arco do Desmatamento, faixa do leste ao sul da região amazônica que recebe maior pressão da fronteira agrícola (BAIMA et al., 2018). Segundo os autores supracitados, rodovias importantes influenciam a pressão por facilitar o deslocamento e escoamento de produção agropecuária, a região da BR-163 (Cuiabá-Santarém) concentra a maior parte das UCs ameaçadas no Pará.

Dessa forma, UCs nas proximidades de estradas são muito mais pressionadas que as distantes, a RBNSC por exemplo, localizada contígua à BR163, foi a UC de proteção integral com maior desmatamento na Amazônia no período de 2006 a 2017, mais de 167 mil hectares degradados (CARDOSO; SOUZA JR., 2018). O levantamento realizado para a elaboração do Plano de Manejo em 2009 já ressaltava a pressão que a reserva havia sofrendo com o desflorestamento, na grande maioria para a implantação de pastagens, atividade que permanece ocorrendo na área, conforme demonstrado no mapa de uso da terra (Figura 3) (BRASIL, 2009). Além disso, estudos apontam que a RBNSC está dentro da região de compra dos frigoríficos, localizada aos arredores da BR-163, confirmando a atividade pecuária dentro da UC (BAIMA et al., 2018).

De uma forma geral, as áreas protegidas desempenham um papel importante na oferta de serviços ecossistêmicos, conservação da biodiversidade e contribuem para mitigação das mudanças climáticas (NAGENDRA et al., 2013), contudo, dados de Araújo et al. (2017) mostram que algumas UCs da Amazônia não estão cumprindo efetivamente com seus objetivos de criação. Um exemplo é a APA Triunfo do Xingu, situada entre os municípios de São Félix do Xingu e Altamira, a UC mais desmatada da Amazônia principalmente para o desenvolvimento da pecuária. Além dela, a Flona do Jamanxim no município de Novo Progresso é considerada a UC federal mais degradada da Amazônia, pressionada e ameaçada por interesses fundiários e da pecuária (ARAÚJO et al., 2017). Ambas as unidades de conservação 
estão localizadas próximas à Rebio, nas proximidades da BR-163, sendo ameaçadas principalmente pela agropecuária.

Embora a atividade agropecuária ser considerada ilegal dentro de unidades de conservação de proteção integral, ainda assim possui potencial de fornecimento de serviços ecossistêmicos relacionado principalmente na provisão de alimentos. Além dessa, a classe "rio, lago e oceano" também disponibiliza bens e serviços à população, visto que especificamente na RBNSC possui grande relevância de provisão de SEs pelo fato da reserva compreender muitas nascentes, formadoras dos rios das bacias do Xingu e do Tapajós, conforme descreve o Plano de Manejo (BRASIL, 2009).

Alguns estudos se mostraram em consonância com os resultados aqui encontrados, a exemplo de Zhang e Ramírez (2019) que utilizou um método para planejamento da infraestrutura verde urbana baseado na capacidade de oferta de SEs em Barcelona, situado na Espanha, onde áreas artificiais com intervenção humana possuem nenhuma ou pouca capacidade de prover serviços ecossistêmicos, corroborando também com resultados encontrados por Sohel et al. (2015) sobre o Parque Nacional de Lawachara e sua área circundante, localizado em Bangladesh, que utilizaram a atribuição de pesos e destacaram que áreas menos perturbadas e mais naturais são importantes fornecedoras de múltiplos SEs, como por exemplo as florestas. Milheiras e Mace (2019), Silva et al. (2017) e Depellegrin et al. (2016) mostram que apesar das áreas cultivadas apresentarem mais serviços de provisão, resultam na diminuição da disponibilidade de determinadas classes de SEs, como os serviços de regulação e culturais, como qualidade do ar e água, e ciclagem de nutrientes, a partir das alterações identificadas no estudo. Importante enfatizar que essas atividades são proibidas dentro dessa categoria de UC.

Da mesma forma ocorreu no mapeamento da RBNSC, que mostrou maior fragilidade nos serviços de regulação e culturais (Figura 5), demonstrando mais sensível às mudanças do uso e ocupação do solo e consequentemente apresentando menor capacidade de oferta. Tal fato provavelmente está relacionado às alterações no meio, tornando a área menos atrativa quanto à informação estética, recreação, outros. Além disso, por estarem menos protegidos pela cobertura vegetal, essas áreas ficam muitos mais susceptíveis às variações climáticas, erosão do solo, a perda da regulação hídrica, diminuição do estoque de carbono e à 
diminuição da ciclagem de nutrientes (MARKEWITZ et al., 2004; ZIMMERMAN et al., 2006; SOLAR et al., 2016).

Zhang e Ramírez (2019) propõem uma classificação em seu estudo, incluindo "área protegida prioritária", as que fornecem maior variedade de serviços ecossistêmicos, geralmente com áreas verdes de alta qualidade, que proveem habitat para espécies animais, proporciona melhorias na qualidade do ar, entre outros benefícios. Nesse sentido, considerando a área aqui estudada, observa-se que outras pesquisas também reforçam a importância da manutenção de áreas detentoras de múltiplos serviços ecossistêmicos (áreas protegidas) e seus reais benefícios. Sohel et al. (2015) contribuem afirmando que o aumento de áreas florestadas pode aumentar ainda a integridade ecológica da área, levando à oferta de outros serviços ecossistêmicos para a região.

Chen et al. (2019) afirmam que as mudanças no uso do solo como a expansão de áreas urbanas e cultivadas resultam em significativas alterações nos SEs, sendo necessário o controle dessas mudanças como forma de melhorar as funções ecossistêmicas. Ainda segundo estes autores, 0 desenvolvimento econômico acarreta na intensificação dessas mudanças, causando degradação nos ecossistemas. Logo, análises da relação de uso e ocupação da terra e serviços ecossistêmicos fornecem fortes evidências acerca da necessidade de aplicação de usos sustentáveis, gestão eficiente das áreas, desenvolvimento econômico e proteção do meio ambiente.

Em relação à metodologia de elaboração de matriz baseada nas classes de uso da terra, Hou et al. (2013) e Jacobs et al. (2015) afirmam que tem se mostrado com um método rápido e válido para medir a oferta dos serviços ecossistêmicos na Europa e Ásia. Kokkoris et al. (2018) adicionam que essa abordagem se mostra relevante na identificação de SEs, produção de informação científica e principalmente destaca a importância de áreas que não vem sendo protegidas de forma eficaz.

\section{Considerações finais}

A Reserva Biológica Nascentes da Serra do Cachimbo foi caracterizada neste estudo com grande potencial para oferta de todas as classes de SEs, principalmente em áreas naturais preservadas. As áreas modificadas por atividades antrópicas foram identificadas com menor potencial de oferta, sendo basicamente fornecedoras de SEs de provisão e manutenção, destacadas como áreas que necessitam de 
maior atenção para não comprometer ainda mais a dinâmica natural na reserva, especialmente com relação aos serviços de regulação e culturais.

Desta forma, a pesquisa pode ser considerada como um passo inicial no mapeamento e monitoramento dessa unidade de conservação que, apesar de abranger nascentes de importantes corpos hídricos na Amazônia e estar situada em área estratégica para frear o desmatamento nessa região, tem seus serviços ecossistêmicos ameaçados pelas mudanças de uso da terra. Apesar do caráter subjetivo da ponderação dos critérios, o método mostrou-se eficiente na identificação, mapeamento e visualização dos serviços ecossistêmicos, cujas avaliações podem ser complementadas em futuros trabalhos.

Por fim, destaca-se que apesar das atividades agropecuárias fornecerem benefícios econômicos a curto prazo, deve-se repensar sobre os desserviços que essas práticas podem gerar a médio e longo prazo à qualidade de vida das populações e ao ecossistema natural como compactação e empobrecimento dos solos, assoreamento de rios, diminuição da fauna e floral nativa, outros. Assim, sugere-se o aprimoramento da gestão e desenvolvimento de planos de proteção para as áreas identificadas com maior potencial de oferta de SEs dentro da RBNSC, com o desenvolvimento e implementação de planos de fiscalização e restauração das áreas com menor potencial, focando na integralização dessas medidas na atualização do Plano de Manejo e na perspectiva da manutenção efetiva dos serviços ecossistêmicos.

\section{Referências Bibliográficas}

ALKEMADE, R.; BURKHARD, B.; CROSSMAN, N.; NEDKOV, S.; K. PETZ. Quantifying ecosystem services and indicators for science, policy and practice. Special Issue.

Ecological Indicators, v. 37, p. 161-162, 2014.

ARAÚJO, E.; BARRETO, P.; BAIMA, S.; GOMES, M. Unidades de conservação mais desmatadas da Amazônia Legal (2012-2015). Belém. Imazon. 92 pp. 2017. Disponível em: http://imazon.org.br/PDFimazon/Portugues/livros/UCS\%20mais\%20desmatadas\%20Amazon ia_2012-2015.pdf

BAIMA, S.; BARRETO, P.; MANSUR, A. Nosso patrimônio ameaçado: Como as unidades de conservação na Amazônia estão em risco. Dossiê. Imazon. 48 pp. 2018. Disponível em: https://k6f2r3a6.stackpathcdn.com/wp-content/uploads/2019/01/DossieUCS_-Imazon.pdf

BALVANERA, P.; DAILY, G. C.; EHRLICH, P. R.; RICKETTS, T. H.; BAILEY, S. A.; KARK, S.; KREMEN, C.; PEREIRA, H. Conserving biodiversity and ecosystem services. Science, v. 291, n. 5511, p. 2047-2047, 2001.

BRASIL. Lei no 9.985, de 8 de julho de 2000. Institui o Sistema Nacional de Unidades de Conservação da Natureza e dá outras providências. Brasília: DOU de 19/07/2000. 
BRASIL. Decreto Federal s/n de 20 de maio de 2005. Institui a criação da unidade de conservação de proteção integral Reserva Biológica Nascentes da Serra do Cachimbo. Brasília: DOU de 23/05/2005.

BRASIL. Plano de Manejo da Reserva Biológica Nascentes da Serra do Cachimbo. Instituto Chico Mendes de Conservação da Biodiversidade. 2009. Disponível em: http://www.icmbio.gov.br/portal/images/stories/imgs-unidadescoservacao/rebio_nascentes_da_serra_do_cachimbo.pdf

BURKHARD, B.; CROSSMAN, N.; NEDKOV, S.; PETZ, K.; ALKEMADE, R. Mapping and modelling ecosystem services for science, policy and practice. Ecosystem Services, v. 4, p. 1-3, 2013.

BURKHARD, B.; KROLL, F.; MÜLLER, F.; WINDHORST, W. Landscapes' capacities to provide ecosystem services - A concept for land-cover based assessments. Landscape Online, v. 15, p. 1-22, 2009.

BURKHARD, B.; MAES, J. Mapping Ecosystem Services. Pensoft Publishers, Sofia, 377 pp., 2017.

CARDOSO, D.; SOUZA JR, C. Degradação florestal em áreas protegidas. Imazon. Belém. Disponível em:

https://imazon.org.br/PDFimazon/Portugues/outros/OEstadoAPs_DegradacaoFlorestal.pdf

CASTRO, A. S.; ANDRADE, D. C. O custo econômico do desmatamento da Floresta Amazônica brasileira (1988-2014). Perspectiva Econômica, v. 12, n. 1, p. 1-15, 2016.

CHEN, W.; CHI, G.; LI, J. The spatial association of ecosystem services with land use and land cover change at the county level in China, 1995-2015. Science of The Total Environment, v. 669, p. 459-470, 2019.

CNUC. Cadastro Nacional de Unidades de Conservação. 2019. Disponível em:

http://www.mma.gov.br/areas-protegidas/cadastro-nacional-de-ucs/consulta-gerar-relatoriode-uc.html

COSTANZA, R.; D'ARGE, R.; DE GROOT, R.; FARBERK, S.; GRASSO, M.; HANNON, B.; LIMBURG, K.; NAEEM, S.; O'NEILL, R. V.; PARUELO, J.; RASKIN, R. G.; SUTTONKK, P.; VAN DEN BELT, M. The value of the world's ecosystem services and natural capital. Nature, v. 387, p. 253-260, 1997.

CRESPIN, S. J.; SIMONETTI, J. A. Loss of ecosystem services and the decapitalization of nature in El Salvador. Ecosystem Services, v. 17, p. 5-13, 2016.

CROSSMAN, N. D.; BURKHARD, B.; NEDKOV, S. Quantifying and mapping ecosystem services. International Journal of Biodiversity Science, Ecosystem Services and Management, v. 8, n. 1-2, p. 1-4, 2012.

CROSSMAN, N. D.; BURKHARD, B.; NEDKOV, S.; WILLEMEN, L.; PETZ, K.; PALOMO, I., DRAKOU, E. G.; MARTÍN-LOPEZ, B.; MCPHEARSON, T.; BOYANOVA, K.; ALKEMADE, R.; EGOH, B.; DUNBAR, M. B.; MAES, J. A blueprint for mapping and modelling ecosystem services. Ecosystem Services, v. 4, p. 4-14, 2013.

DAILY, G. C. Nature's services: societal dependence on natural systems. Washington DC: Island Press. 1997.

DAL'AASTA, A. P.; AMARAL, S.; SOARES, F. R.; MONTEIRO, A. M. V. Evolução recente da população urbana comparada à evolução do desmatamento nos municípios do Distrito Florestal Sustentável da BR-163 (PA). In: Anais do XVIII Encontro Nacional de Estudos Populacionais. Águas de Lindóia/SP, 2012.

DE GROOT, R. S.; WILSON, M. A., BOUMANS, R. M. J. A typology for the classification, description and valuation of ecosystem function goods and services. Ecological

Economics, v. 41, p. 393-408, 2002. 
DE GROOT, R.S.; FISHER, B.; CHRISTIE, M.; ARONSON, J.; BRAAT, L.; HAINESYOUNG, R.; GOWDY, J.; MALTBY, E.; NEUVILLE, A.; POLASKY, S.; PORTELA, R.; RING, I. Integrating the ecological and economic dimensions in biodiversity and ecosystem service valuation. The Economics of Ecosystems and Biodiversity: Ecological and Economic Foundations. P. Kumar. London, Earthscan, p. 3-40, 2010.

DEPELLEGRIN, D.; PEREIRA, P.; MISIUNÈ, I.; EGARTER-VIGL, L. Mapping ecosystem services potential in Lithuania. International Journal of Sustainable Development \& World Ecology, v. 23, n. 5, p. 441-455, 2016.

GEIJZENDORFFER, I. R.; MARTÍN-LÓPEZ, B.; ROCHE, P. K. Improving the identification of mismatches in ecosystem services assessments. Ecological Indicators, v. 52, p. 320-33, 2015.

GÓMEZ-BAGGETHUN, E.; BARTON, D. N. Classifying and valuing ecosystem services for urban planning. Ecological Economics, v. 86, p. 235-245, 2013.

HEAL, G. M.; BARBIER, E. B.; BOYLE, K. J.; COVICH, A. P.; GLOSS, S. P.; HERSHNER, C. H.; HOEHN, J. P.; PRINGLE, C. M.; POLASKY, S.; SEGERSON, K.; SHRADERFRECHETTE, K. Valuing ecosystem services: toward better environmental decisionmaking. In: Committee on Assessing and Valuing the Services of Aquatic and Related Terrestrial Ecosystems - Water Science and Technology Board - Division on Earth and Life Studies. The National Academies Press, Washington D.C., 291 pp, 2005.

HOU, Y.; BURKHARD, B.; MÜLLER, F. Uncertainties in landscape analysis and ecosystem service assessment. J. Environ. Manage, v. 127, p. 117-131, 2013.

IBGE. Instituto Brasileiro de Geografia e Estatística. 2017. Disponível em: https://downloads.ibge.gov.br/downloads_geociencias.htm

ISA. Instituto Socioambiental. 2019. Disponível em: https://uc.socioambiental.org/

JACOBS, S.; BURKHARD, B.; VAN DAELE, T., STAES, J.; SCHNEIDERS, A. The 'Matrix Reloaded': a review of expert knowledge use for mapping ecosystem services. Ecol. Model., v. 295, p. 21-30, 2015.

KANDZIORA, M.; BURKHARD, B.; MÜLLER, F. Mapping provisioning ecosystem services at the local scale using data of varying spatial and temporal resolution. Ecosystem Services, v. 4, p. 47-59, 2013.

KAUSHAL, S. S.; DUAN, S.; DOODY, T. R.; HAQ, S.; SMITH, R. M.; JOHNSON, T. A. N.; NEWCOMB, K. D.; GORMAN, J.; BOWMAN, N.; MAYER, P. M.; WOOD, K. L.; BELT, K. T.; STACK, W. P. Human-accelerated weathering increases salinization, major ions, and alkalinization in fresh water across land use. Appl. Geochem., v. 83, p. 121-135, 2017.

KOKKORIS, I. P; EVANGELIA, G. D.; MAES, J.; DIMOPOULOS, P. Ecosystem services supply in protected mountains of Greece: setting the baseline for conservation management. International Journal of Biodiversity Science, Ecosystem Services \& Management, v. 14, n. 1, p. 45-59, 2018.

KZAM, A. L.; LOPES, M. L. B.; ABUD, G. B.; CORREA, R. S. S. Fragmentação territorial: análise do plebiscito de divisão do estado do Pará. Boletim de Geografia, v. 36, n. 1, p. 116, 2018.

LE CLEC'H, S.; SLOAN, S.; GOND, V.; CORNU, G.; DECAENS, T.; DUFOUR, S.; GRIMALDI, M.; OSZWALD, J. Mapping ecosystem services at the regional scale: the validity of an upscaling approach. International Journal of Geographical Information Science, v. 32, n. 8, p. 1593-1610, 2018.

LIU, J.; JIANG, L.; GAO, Z.; MIN, Y.; QIN, K.; YANG, X. Ecosystem services insights into water resources management in China: a case of Xi'an City. Int. J. Environ. Res. Public Health, v. 13, n. 12, p. 1169-1188, 2016. 
MAES, J.; PARACCHINI, M. L.; ZULIAN, G. Towards an atlas of ecosystem services. A European assessment of the provision of ecosystem services, JRC Scientific and Technical Reports. 2011. 88 pp.

MALINGA, R.; GORDON, L. J.; JEWITT, G.; LINDBORG, R. Mapping ecosystem services across scales and continents - A review. Ecosystem Services, v. 13, p. 57-63, 2015.

MARKEWITZ, D; DAVIDSON, E; MOUTINHO, P; NEPSTAD, D. Nutrient loss and redistribution after forest clearing on a highly weathered soil in Amazonia. Ecological Applications, v. 14, n. 4, p. 177-199, 2004.

MAPBIOMAS. Projeto de Mapeamento Anual da Cobertura e Uso do Solo do Brasil. Coleção 3.0 da Série Anual de Mapas de Cobertura e Uso de Solo do Brasil. 2019. Disponível em: http://mapbiomas.org/map\#coverage

MEA. Millennium Ecosystem Assessment. Ecosystems and Human well-being. Synthesis. Island Press, Washington, DC. 2005. 155 p.

MILHEIRAS, S. G.; MACE, G. M. Assessing ecosystem service provision in a tropical region with high forest cover: Spatial overlap and the impact of land use change in Amapá, Brazil. Ecological Indicators, v. 99, p. 12-18, 2019.

MONTEIRO, S. M. C. Reflexões jurídico-institucionais a respeito do território da Flona do Jamanxim: avanços, recuos e conflitos atuais. Revista Ciências da Sociedade (RCS), v. 2, n. 3, p. 281-300, 2018.

NAGENDRA, H.; LUCAS, R.; HONRADO, J. P.; JONGMAN, R. H. G.; TARANTINO, C.; ADAMO, M.; MAIROTA, P. Remote sensing for conservation monitoring: assessing protected areas, habitat extent, habitat condition, species diversity, and threats. Ecological Indicators, v. 33, p. 45-59, 2013.

RAMIREZ-GOMEZ, S. O. I.; TORRES-VITOLAS, C. A.; SCHRECKENBERG, K.; HONZÁK, M.; CRUZ-GARCIA, G. S.; WILLCOCK, S.; PALACIOS, E.; PÉREZ-MIÑANA, E.; VERWEIJ, P. A.; POPPY, G. M. Analysis of ecosystem services provision in the Colombian Amazon using participatory research and mapping techniques. Ecosystem Services, v. 13, p. 93107, 2015.

SOHEL, M. S. I.; MUKUL, S. A.; BURKHARD, B. Landscape's capacities to supply ecosystem services in Bangladesh: A mapping assessment for Lawachara National Park.

Ecosystem Services, v. 12, p. 128-135, 2015.

SILVA, M. M.; OLIVEIRA, F. A.; SANTANA, A. C. Mudanças na dinâmica de uso das florestas secundárias em Altamira, Estado do Pará, Brasil. Rev. Cienc. Agrar., v. 58, n. 2, p. 176-183, 2015.

SILVA, M. M.; OLIVEIRA, F. A.; SANTANA, A. C. Mudanças socioambientais no uso da terra em Altamira, Amazônia oriental. Novos Cadernos - NAEA, v. 20, n. 3, p. 181-202, 2017.

SOLAR, R. R. D. C.; BARLOW, J.; ANDERSEN, A. N.; SCHOEREDER, J. H.;

BERENGUER, E.; FERREIRA, J. N.; GARDNER, T. A. Biodiversity consequences of landuse change and forest disturbance in the amazon: A multi-scale assessment using ant communities. Biological Conservation, v. 197, p. 98-107, 2016.

TEEB. The Economics of Ecosystems and Biodiversity. Integrando a Economia da Natureza: uma síntese da abordagem, conclusões e recomendações do TEEB. 2010.

TROY, A.; WILSON, M. A. Mapping ecosystem services: practical challenges and opportunities in linking GIS and value transfer. Ecological Economics, v. 60, p. 435-449, 2006.

TUPIASSU, L., JEAN-RAPHAËL, G., FADEL, L. P. S. L. O impacto do ICMS verde nos municípios prioritários do estado do Pará. Revista de Estudos Empíricos em Direito, v. 5, n. 2, p. 67-86, 2018. 
WOLFF, S.; SCHULP, C. J. E.; VERBURG, P. H. Mapping ecosystem services demand: A review of current research and future perspectives. Ecological Indicators, v. 55, p. 159$171,2015$.

YOUNG, C. E. F.; MEDEIROS, R. Quanto vale o verde: a importância econômica das unidades de conservação brasileiras. Rio de Janeiro: Conservação Internacional, 180pp, 2018.

ZHANG, S.; RAMÍREZ, F. M. Assessing and mapping ecosystem services to support urban green infrastructure: The case of Barcelona, Spain. Cities, v. 92, p. 59-70, 2019.

ZIMMERMANN, B.; ELSENBEER, H.; MORAES, J. M. The influence of land-use changes on soil hydraulic properties: implications for runoff-generation. Forest Ecology and Management, v. 222, n. 1-3, p. 29-38, 2006.

\section{NOTAS DE AUTOR}

CONTRIBUIÇÃO DE AUTORIA

Mayara Gomes da Silva - Concepção. Coleta de dados, Análise de dados, Elaboração do manuscrito e aprovação da versão final do trabalho.

Norma Ely Santos Beltrão - Concepção e elaboração do manuscrito, Participação ativa da discussão dos resultados, Revisão e aprovação da versão final do trabalho.

Gundisalvo Piratoba Morales - Concepção, revisão, Participação da discussão dos resultados e aprovação da versão final do trabalho.

\section{FINANCIAMENTO}

Coordenação de Aperfeiçoamento de Pessoal de Nível Superior - CAPES pela bolsa de mestrado concedida ao autor M.G.S.

\section{CONSENTIMENTO DE USO DE IMAGEM}

Não se aplica

\section{APROVAÇÃO DE COMITÊ DE ÉTICA EM PESQUISA}

Não se aplica

\section{CONFLITO DE INTERESSES}

Não se aplica

\section{LICENÇA DE USO}

Este artigo está licenciado sob a Licença Creative Commons CC-BY-NC. Com essa licença você pode compartilhar, adaptar, criar para qualquer fim, sem uso comercial e desde que atribua a autoria da obra.

\section{HISTÓRICO}

Recebido em: 28-01-2020

Aprovado em: 11-11-2020 